\title{
Asymptotically free models and discrete non-Abelian groups
}

\author{
Sergio Caracciolo \\ Scuola Normale Superiore, INFM and INFN - Sezione di Pisa \\ I-56100 Pisa, ITALIA \\ Internet: Sergio.Caracciolo@sns.it \\ Andrea Montanari \\ Laboratoire de Physique Théorique de l'Ecole Normale Supérieure* \\ 24, rue Lhomond, 75231 Paris CEDEX 05, FRANCE \\ Internet: Andrea.Montanari@lpt.ens.fr \\ Andrea Pelissetto \\ Dipartimento di Fisica and INFN - Sezione di Roma I \\ Università degli Studi di Roma "La Sapienza" \\ I-00185 Roma, ITALIA \\ Internet: Andrea.Pelissetto@roma1.infn.it
}

October 27, 2018

\begin{abstract}
We study the two-dimensional renormalization-group flow induced by perturbations that reduce the global symmetry of the $O(3) \sigma$-model to the discrete symmetries of Platonic solids. We estimate the value of the correlation length at which differences in the behaviour of the various models should be expected. For the icosahedron model with nearest-neighbor interactions, we find $\xi \gtrsim 200$. We provide an explanation for the recent numerical results of Patrascioiu and Seiler and of Hasenfratz and Niedermayer.
\end{abstract}

*UMR 8549, Unité Mixte de Recherche du Centre National de la Recherche Scientifique et de l' Ecole Normale Supérieure. 


\section{Introduction}

Quantum field theories with a non-Abelian continuous symmetry group of invariance play a major role both in particle and in condensed-matter physics. Two cases are of particular interest: two-dimensional spin models with non-Abelian global symmetry group, and four-dimensional gauge theories with local non-Abelian gauge invariance. According to the common wisdom these two cases share the peculiar feature of asymptotic freedom (AF).

In the lattice formulation it is quite easy to replace the continuous group by one of its discrete subgroups. In this case, due to the discreteness of the group, the action has a finite gap and at least a freezing transition is expected. N Nonetheless, at large enough temperature one expects only small differences and indeed, finite subgroups have been used in Monte Carlo updates to simulate continuous groups [2, 3].

When the symmetry group is Abelian it may happen that the discrete symmetry is enlarged to a continuous one. The massless intermediate phase of $Z(N)$ models with

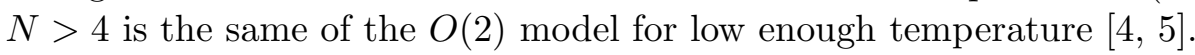

It has also been suggested that a similar phenomenon occurs in non-Abelian models. Patrascioiu and Seiler [6, 7, 8, 9] have often criticized the conventional wisdom on AF for the continuous group and proposed an alternative scenario in which a low-temperature massless phase appears. If this possibility really happens it is plausible to accept the idea that, for example, in $d=2$ the $O(3)$ model is in the same universality class of the dodecahedron spin model.

In order to test this conjecture several large-scale simulations have been performed [10, 11, 8]. In particular, the finite-size scaling curve for the second-moment correlation length measured in 112, 13 and the renormalized coupling for the $O(3)$ model have been compared with the results obtained for discrete spin models with different discrete subgroups and nearest-neighbor interactions. It was found that the icosahedron model and the $O(3)$ model are practically indistinguishable at present-day values of $\xi$. This is not totally surprising: after all, for $\beta$ small enough one expects only tiny differences since in the presence of large fluctuations the discreteness of the spin space should not play an important role (this was indeed the motivation of Refs. [2], 3]). However, what is more surprising is that the discrepancy seems to decrease as $\beta$ increases (see the results of Ref. [10] for the renormalized zero-momentum four-point coupling), while naively one would have expected the opposite.

These numerical results have been interpreted as evidence that the $O(3)$ and the icosahedron model have the same continuum limit. In this paper, we will show that this conclusion is in contrast with the common theoretical understanding of the $O(3)$ model: If the continuum limit of the $O(3) \sigma$ model is correctly described by the perturbative renormalization group (RG), then the icosahedron and the $O(3)$ model belong to different universality classes, contrary to what suggested in Refs. [10, 11].

For this purpose, we study the effect of perturbations that break the $O(3)$ symmetry down to a discrete subgroup and show that any such perturbation is a relevant perturbation that modifies the universal behavior. A similar analysis for the cubic symmetry was performed by Pelcovits and Nelson [14] and in the context of the $X Y$ model in Ref. [4. We then try to provide an explanation to the numerical data. We analyze a model

\footnotetext{
${ }^{1}$ In some models, for instance in some $Z(N)$ models, between the ordered and the disordered phase, there may also be an intermediate massless phase [1].
} 
with icosahedral symmetry that interpolates between the nearest-neighbor $O(3)$ model $\left(h_{6}=0\right)$ and the standard icosahedron model $\left(h_{6}=+\infty\right)$, using perturbation theory. When $h_{6}$ is small, we find for $\xi \lesssim 200$ a behavior that is similar to that observed numerically: the difference between the icosahedral and the $O(3)$ model decreases as $\beta$ increases. However, this apparent convergence is misleading, because for $\xi \gtrsim 200$ the difference between the two models increases as $\beta$ increases, with the result that the two models do not lie in the same universality class. On the basis of these results we predict that the difference between the standard icosahedron model considered in [10, 11, 8] and the $O(3)$ model should probably become visible only for $\xi \gtrsim 200$.

We stress that all our arguments assume the validity of the perturbative RG for the $O(3)$ model. If this is not correct, it is possible that the icosahedron and the $O(3)$ models have the same universal behavior as conjectured by Patrascioiu and Seiler.

\section{Renormalization-group analysis of perturba- tions with discrete symmetry}

In this Section we want to perform a RG analysis of the discrete models. For this purpose we consider the lattice Hamiltonian

$$
\beta H^{\mathrm{latt}}=\frac{\beta}{2} \sum_{x \mu}\left(\Delta_{\mu} \boldsymbol{\sigma}\right)^{2}-h_{n} \sum_{x} I_{n}\left(\boldsymbol{\sigma}_{x}\right),
$$

where $\boldsymbol{\sigma}_{x}$ is a unit vector in $\mathbb{R}^{3}$,

$$
\Delta_{\mu} \boldsymbol{\sigma}_{x}=\boldsymbol{\sigma}_{x+\mu}-\boldsymbol{\sigma}_{x}
$$

and $\mu=1,2$ are the positive directions on a square lattice with lattice spacing $a=1$. Here, $I_{n}\left(\boldsymbol{\sigma}_{x}\right)$ is a polynomial of $\boldsymbol{\sigma}_{x}$ that is invariant under the action of the discrete group and belongs to an irreducible representation of the $O(3)$ group, i.e. it has a well-defined $O(3)$ spin $n$. Such polynomials can be found for all discrete subgroups that correspond to Platonic solids. The lowest-rank tensors for each subgroup are obtained in App. A.2. For the tetrahedron, the cube (or the octahedron), and for the icosahedron (or the dodecahedron), the lowest-rank invariant tensors have spin $n=3$, 4, and 6. Explicitly, we have for the three cases respectively

$$
\begin{aligned}
& I_{3}=-i 3 \sqrt{\frac{2 \pi}{35}}\left(Y_{3,2}-Y_{3,-2}\right), \\
& I_{4}=-\sqrt{\pi}\left[Y_{4,0}+\sqrt{\frac{5}{14}}\left(Y_{4,4}+Y_{4,-4}\right)\right], \\
& I_{6}=\sqrt{\frac{4 \pi}{13}}\left[Y_{6,0}-\sqrt{\frac{7}{11}}\left(Y_{6,5}-Y_{6,-5}\right)\right] .
\end{aligned}
$$

Here, we have introduced the spherical harmonics

$$
Y_{l, m}(\theta, \phi)=\sqrt{\frac{2 l+1}{4 \pi} \frac{(l-m) !}{(l+m) !}} P_{l, m}(\cos \theta) e^{i m \phi},
$$


where $P_{l, m}(x)$ are the associated Legendre functions, and we have written the threedimensional spin $\boldsymbol{\sigma}$ in terms of polar coordinates $\theta$ and $\phi$. These tensors are not unique, since the normalization is arbitrary and they are defined modulo a rotation. [f For the purpose of showing that discrete-subgroup perturbations are relevant, the value of $n$ plays no role and one can use any invariant tensor. One could also consider tensors that do not have definite spin and are sums of invariant $O(3)$-irreducible tensors. This would only make the discussion more cumbersome without changing the physical results.

The partition function is defined as usual by

$$
Z=\int e^{-\beta H^{l a t t}} \prod_{x} \mathrm{~d}^{N} \boldsymbol{\sigma}_{x} \delta\left(\boldsymbol{\sigma}_{x}^{2}-1\right)
$$

The Hamiltonian (11) interpolates between the $O(3)$ model $\left(h_{n}=0\right)$ and the discretesymmetry model $\left(\left|h_{n}\right|=\infty\right)$.

If, as claimed in Refs. 10, 11, the discrete-symmetry model is also AF, in the RG language, this means that the added term is an irrelevant perturbation. Let us perform a standard RG calculation around the theory with $h_{n}=0$. If $G^{(p)}\left(k_{1}, \ldots, k_{p} ; \beta, h_{n}\right)$ is the connected $p$-point correlation function, we can perform an expansion in powers of $h_{n}$, i.e. rewrite

$$
G^{(p)}\left(k_{1}, \ldots, k_{p} ; \beta, h_{n}\right)=\sum_{q=0} \frac{1}{q !} h_{n}^{q} G^{(p, q)}\left(k_{1}, \ldots, k_{p} ; 0, \ldots, 0 ; \beta, 0\right),
$$

where $G^{(p, q)}\left(k_{1}, \ldots, k_{p} ; l_{1}, \ldots, l_{q} ; \beta, 0\right)$ is the correlation function with $p$ fields and $q$ insertions of the breaking operator at momenta $l_{1}, \ldots, l_{q}$, computed for $h_{n}=0$, i.e. in the $O(3)$-model. Such correlation functions cannot be computed directly in perturbation theory for $l_{1}=\ldots=l_{q}=0$, since the momenta of the insertions are zero and perturbative expansions 9 are valid only for large momenta, i.e. for distances much smaller than the correlation length. Indeed, we are expanding around an ordered configuration and this is correct only for $|x| \ll \xi$ because of the Mermin-Wagner theorem. However, within the usual theoretical framework, there is a standard way out. Consider $G^{(p, q)}\left(k_{1}, \ldots, k_{p} ; l_{1}, \ldots, l_{q} ; \beta, 0\right)$ in the perturbative regime (large momenta) and use perturbation theory to derive the $\mathrm{RG}$ equation

$$
\left[-a \frac{\partial}{\partial a}+W(t) \frac{\partial}{\partial t}+q \gamma^{(n)}(t)+\frac{p}{2} \gamma(t)\right] G^{(p, q)}\left(k_{1}, \ldots, k_{p} ; l_{1}, \ldots, l_{q} ; \beta, 0\right)=0,
$$

\footnotetext{
${ }^{2}$ In Eq. (3) we have defined $I_{n}$ so that each polynomial has maximal value one on the vertices of the tetrahedron, cube, and icosahedron respectively. The minimal value (maximal in absolute value) of $I_{4}$ on the vertices of the octahedron is $-3 / 2$ and of $I_{6}$ on the vertices of the dodecahedron is $-5 / 9$. Of course, the normalization of $I_{n}$ is irrelevant for our discussion.

${ }^{3}$ Note that, if a discrete model is obtained fo $h_{n}=+\infty$, the dual model (see App. A.1 for the definition of duality) is obtained for $h_{n}=-\infty$.

${ }^{4}$ Strictly speaking, the claim has been made only for $\left|h_{n}\right|=\infty$. However, it is difficult to imagine a scenario in which the theory is not $O(3)$ invariant for $h_{n}$ small and recovers the continuous invariance by increasing the strength of the discrete-symmetry term.

${ }^{5}$ Even for large momenta, perturbative expansions are well-defined only for $O(3)$-invariant quantities. Thus, in order to have an infrared-finite perturbation theory one must rewrite each correlation function in terms of $O(3)$-invariant expressions. For instance, to compute correlations of $I_{n}$ we can use the identity $\left\langle Y_{l_{1}, m_{1}}\left(\boldsymbol{\sigma}_{0}\right) Y_{l_{2}, m_{2}}\left(\boldsymbol{\sigma}_{x}\right)\right\rangle=\delta_{l_{1}, l_{2}} \delta_{m_{1}, m_{2}}\left\langle P_{l_{1}}\left(\boldsymbol{\sigma}_{0} \cdot \boldsymbol{\sigma}_{x}\right)\right\rangle /(4 \pi)$, where $P_{l}(x)$ is a Legendre polynomial. The righthand side is infrared-finite.
} 
where $t \equiv 1 / \beta, W(t), \gamma^{(n)}(t)$, and $\gamma(t)$ are respectively the lattice $\beta$-function and the lattice anomalous dimensions of $I_{n}$ and of the field. The crucial assumption is that this equation is valid for all momenta, even outside the strictly perturbative region.6

Taking into account the scaling dimension of the correlation function, at zero external momenta $\left(k_{i}=l_{i}=0\right)$, we have

$$
G^{(p, q)}(0 ; 0 ; \beta, 0)=A^{(p, q)} G^{(p, 0)}(0 ; \beta, 0) \exp \left[q \int_{t_{0}}^{t} \frac{2-\gamma^{(n)}(s)}{W(s)} d s\right],
$$

where $A^{(p, q)}$ is a non-perturbative constant. Therefore, we obtain finally

$$
G^{(p)}\left(0 ; \beta, h_{n}\right)=G^{(p)}(0 ; \beta, 0) \sum_{q=0} \frac{1}{q !} A^{(p, q)} h_{n}^{q} \exp \left[q \int_{t_{0}}^{t} \frac{2-\gamma^{(n)}(s)}{W(s)} d s\right] .
$$

By using the perturbative RG, we have been able to factor out the $h$ dependence of the correlation function. Now, for $t \rightarrow 0$ we find immediately

$$
G^{(p)}\left(0 ; \beta, h_{n}\right) \approx G^{(p)}(0 ; \beta, 0) \sum_{q=0} \frac{1}{q !} A^{(p, q)}\left(h_{n} t^{\rho_{n}} \exp \left[\frac{4 \pi}{t}\right]\right)^{q},
$$

where $\rho_{n}$ is an easily computable exponent. The correction term diverges for $t \rightarrow 0$, showing that the breaking term is a relevant interaction in the RG sense.

Equivalently, one can imagine of considering a scale-dependent renormalized coupling $h_{n}(s)$. Then, the RG flow has the form

$$
\frac{1}{h_{n}(s)} \frac{d h_{n}(s)}{d s}=2-\gamma^{(n)}[t(s)]
$$

where $\exp (-s)$ is the change of the scale and $t(s)$ is the running coupling constant. Since $\gamma^{(n)}(t)$ vanishes for $t \rightarrow 0$, any perturbation of this type is relevant in the continuum limit. We would like to point out that this is not unexpected. Since in two dimensions the field $\boldsymbol{\sigma}$ is dimensionless, any polynomial in $\boldsymbol{\sigma}$ is a relevant operator.

Equations (9) and (10) deserve some additional comments. First of all, they give an expansion of $G^{(p)}\left(0 ; \beta, h_{n}\right)$ in powers of the scaling variable

$$
z \equiv h_{n} t^{\rho_{n}} \exp (4 \pi / t)
$$

and are therefore valid only for $z \ll 1$. However, for our purposes, the only relevant information we obtain from Eq. (10) is that the correct scaling variable is $z$, i.e., that we can define the limit $t \rightarrow 0, h_{n} \rightarrow 0$ at fixed $z$, obtaining

$$
G^{(p)}\left(0 ; \beta, h_{n}\right)=G^{(p)}(0 ; \beta, 0) \Phi^{(p)}(z)
$$

where $\Phi^{(p)}(z)$ is a nonperturbative crossover function. Eq. (13) can be derived directly from the RG equation

$$
\left[-a \frac{\partial}{\partial a}+W(t) \frac{\partial}{\partial t}+\gamma^{(n)}(t) h_{n} \frac{\partial}{\partial h_{n}}+\frac{p}{2} \gamma(t)\right] G^{(p)}\left(k_{1}, \ldots, k_{n} ; \beta, h_{n}\right)=0,
$$

\footnotetext{
${ }^{6}$ The correlation functions are computable in perturbation theory if we introduce an infrared cutoff, for instance if we work in a finite volume. However, they will be infrared divergent, so that one cannot take the infinite-volume limit naively. In the standard theoretical framework, one uses the perturbative expressions to derive an RG equations and then assumes the such equations are also satisfied by the infinite-volume quantities. Whatever the procedure, the result is identical.
} 
which we assume to be valid for all momenta in a neighborhood of $h_{n}=t=0$. Of course, we can only compute the scaling behavior of the correlation functions, but not their explicit expressions. Our ignorance of the long-wavelength physics is encoded here in the nonperturbative nature of the function $\Phi^{(p)}$.

Our result (13) provides what in statistical mechanics is called a crossover scaling function for a fixed point perturbed by two relevant interactions (or, in this case, by one relevant interaction $h_{n}$ and one marginally relevant interaction $\left.t\right)$ : namely, it gives the leading behavior in the limit $t \rightarrow 0, h_{n} \rightarrow 0$ with $z$ fixed. Note that Eq. (13) can also be used to predict the behavior of the phase-transition line for $h_{n}$ small [15, 16], if such a transition exists. Indeed, if $\beta_{c}\left(h_{n}\right)$ is the critical point of the theory at fixed $h_{n}$, for $h_{n}$ small we should have, for $h_{n}$ small,

$$
h_{n}=z^{*} \beta_{c}\left(h_{n}\right)^{\rho_{n}} \exp \left[-4 \pi \beta_{c}\left(h_{n}\right)\right]
$$

where $z^{*}$ is a nonperturbative constant.

We want now to explain the numerical results of Refs. [10, 11, 9], who found that the difference in behavior between the standard $O(3)$ model and the discrete model was decreasing as $\beta$ increased. A possible explanation of this phenomenon is that the RG flux first reduces the size of the perturbation which then increases again as $\beta$ increases. Since in the high-temperature regime, one expects indeed the two models to be quite similar, this could explain the fact that they are numerically indistinguishable at the values of $\beta$ that can be simulated today.

To make this picture more quantitative, let us consider the Hamiltonian (1) with $h_{n}$ and $z$ small, so that we can use Eq. (9). Then, suppose that there exists $t^{\text {eff }}=1 / \beta^{\text {eff }}$ such that $\gamma^{(n)}\left(t^{\text {eff }}\right)=2$ and $2-\gamma^{(n)}\left(t^{\text {eff }}\right)<0$ for $\beta<\beta^{\text {eff }}$. In this case, Eq. (9) would predict the following behavior. For $\beta$ small, the difference would apparently decrease as $\beta$ increases, which could seem to indicate that the interaction is irrelevant. However, as soon as $\beta$ becomes larger than $\beta^{\text {eff }}$ the discrepancy starts increasing again. Now, notice that the discrete model, in the vicinity of the $O(3)$ fixed point, will generate perturbations of arbitrary spin. However, $\gamma^{(n)}$ increases with $n$, and thus the most relevant perturbation is associated to $I_{n}$ with the smallest possible value of $n$. Therefore, we should consider $n=3,4,6$ for the tetrahedron, the cube and the octahedron, the dodecahedron and the icosahedron respectively.

We can try to evaluate $\beta^{\text {eff }}$ by using the perturbative expressions for the anomalous dimension of $I_{n}$ in the $O(3)$ nearest-neighbor lattice model. Explicit three-loop expressions are reported in App. B. We obtain the following estimates

$$
\begin{aligned}
& \beta^{(3), \mathrm{eff}}=0.75 \\
& \beta^{(4), \mathrm{eff}}=1.08 \\
& \beta^{(6), \mathrm{eff}}=1.95
\end{aligned}
$$

which are quite stable with respect to the loop order. Thus, we expect that for $\beta<$ $\beta^{(i)}$, eff the breaking to the corresponding subgroup of $O(3)$ appears as irrelevant. Now,

\footnotetext{
${ }^{7}$ Eq. (13) is valid for any perturbation and any $n$, even if it is not associated to a discrete group. For instance, one can take $n=1$ and $I_{1}(\boldsymbol{\sigma})=\cos \theta$, obtaining the usual magnetic perturbation. In this case, Eq. (13) is the usual crossover equation (for $p=1$ it is usually called equation of state) reported in textbooks, see, e.g., Ref. [17]. Instead, Eq. (15) that will be derived below makes no sense since at fixed $h_{1}$ there is no phase transition.
} 
$\beta \approx 1$ corresponds to a very small correlation length. Therefore, as soon as $\xi>1$, one immediately realizes that the tetrahedron and the cubic model are not asymptotically free. On the other hand, $\beta=1.95$ corresponds to a quite large value of the correlation length $\xi=166.7(4)$ 12]. Therefore, we expect the discrepancy to decrease steadily as $\beta$ increases, till values of $\xi$ of order 200 and increase steadily afterwards. Thus, a clear signal of the difference between the two models requires simulations on quite large lattices with $\xi \gg 200$.

It is important to notice that these estimates are valid for perturbations of the lattice nearest-neighbor $O(3)$ action only. Indeed, as it should be expected, $\beta^{(n) \text {,eff }}$ is nonuniversal, being the solution of $\gamma^{(n)}\left(t^{\text {eff }}\right)=2$, where $\gamma^{(n)}(t)$ is the action-dependent anomalous dimension of $I_{n}$. This means that, by changing the action, it is well possible that the system shows a non- $O(3)$-invariant behavior for much smaller values of $\xi$.

The argument given here applies quantitatively only for $h_{n}$ small. Nonetheless, for the discrete models considered in the simulations it represents a plausible scenario which explains the numerical results and is compatible with the standard theoretical framework used in the analyses of the critical behavior of the $O(3)$ model. Indeed, since all simulations are performed in the region in which $1<\xi \lesssim 100$, it predicts that the cubic and the tetrahedron model are clearly different from the $O(3)$ model, while the icosahedron results should mimic the $O(3)$ ones.

It is interesting to notice that the values of $\beta^{(n) \text {,eff }}$ are close to the critical value for each discrete model $\beta_{c}^{(n)}$. For instance, since the tetrahedron model is equivalent to the 4 -state Potts model with $\beta=3 / 4 \beta_{\text {Potts }}$ we have

$$
\beta_{c}^{(3)}=\frac{3}{4} \log 3 \approx 0.82,
$$

which is only slightly higher than (16). Analogously, the cubic model is equivalent to the product of three Ising models with $\beta=3 \beta_{\text {Ising }}$ so that

$$
\beta_{c}^{(4)}=\frac{3}{2} \log (1+\sqrt{2}) \approx 1.32 .
$$

There is also 18 a numerical estimate for $\beta_{c}^{(6)} \approx 2.15$. Note also that $\beta^{(n) \text {, eff }}<\beta_{c}^{(n)}$, an inequality which shows that our $\beta^{(n) \text {, eff }}$ correspond always to temperatures above the freezing transition.

\section{Acknowledgments}

A.M. thanks E. Brézin for a stimulating conversation on the subject of this paper. We also thank A. D. Sokal for a careful reading of our manuscript, P. Hasenfratz and F. Niedermayer for many useful comments.

\section{A Discrete subgroups of $O(3)$}

\footnotetext{
${ }^{8}$ This number is simply an order of magnitude. Indeed, even if our estimates are quite stable with respect to $\beta$, the correlation length varies rapidly with $\beta$. For instance, for $\beta=1.90$ (resp. 2.00), which are very close to our estimate of $\beta^{(6) \text {,eff }}$ we have $\xi=122.3(3)(228.5(7)$ resp.) [12].
} 


\section{A.1 The Platonic solids}

The icosahedron and the dodecahedron are two Platonic solids. They are regular convex polyhedra [19], with regular and equal faces, and are such that each vertex belongs to the same number of edges. If its faces are $p$-gons (polygons with $p$ sides), $q$ of them surrounding each vertex, the polyhedron is denoted by $\{p, q\}$. The possible values for $p$ and $q$ may be enumerated as follows. The solid angle at a vertex has $q$ face-angles, each $(p-2) \pi / p$. Of course, the sum of these $q$ angles must be less than $2 \pi$. Therefore, we have

$$
\frac{1}{q}+\frac{1}{p}>\frac{1}{2}
$$

Thus, $\{p, q\}$ cannot have any other values than

$$
\{3,3\},\{3,4\},\{4,3\},\{3,5\},\{5,3\} .
$$

They correspond to the tetrahedron, the octahedron, the cube, the icosahedron, and the dodecahedron.

Consider the regular polyhedron $\{p, q\}$ with its $N_{0}$ vertices, $N_{1}$ edges, and $N_{2}$ faces, where $N_{0}-N_{1}+N_{2}=2$ by Euler's formula. As each face touches $p$ edges and each edge belongs to 2 faces, then

$$
p N_{2}=2 N_{1} \text {. }
$$

Analogously, since each vertex belongs to $q$ edges and each edge touches 2 vertices, we obtain

$$
q N_{0}=2 N_{1}
$$

These relations and Euler's formula imply

$$
\frac{1}{q}+\frac{1}{p}-\frac{1}{2}=\frac{1}{N_{1}}
$$

which offers a quantitative evaluation to the inequality (21).

Consider now the sphere which touches all the edges. If we replace each edge by a perpendicular line touching the sphere at the same point, we obtain the $N_{1}$ edges of the dual polyhedron $\{q, p\}$ which has $N_{2}$ vertices and $N_{0}$ faces.

We are interested in the rotation groups of the regular polyhedra. They are finite groups, so that every rotation must have an angle commensurable with $\pi$. In fact, the smallest angle of rotations around a given axis is a submultiple of $2 \pi$, and all other angles of rotation about the same axis are multiples of the smallest one. If $2 \pi / k$ is the smallest angle, then the rotations about this axis form a cyclic group of order $k$, and one speaks of an axis of $k$-fold rotation.

Two dual polyhedra have the same rotation group. The center of the polyhedron $\{p, q\}$ is joined to the vertices, mid-edge points, and centers of faces, by axes of $q$-fold, 2 -fold, and $p$-fold rotation. But the vertices, mid-edge points, and centers of faces occurs in antipodal pairs. Hence, the total number of rotations, excluding the identity, is

$$
\frac{1}{2}\left[(q-1) N_{0}+N_{1}+(p-1) N_{2}\right]=2 N_{1}-1
$$

so that the order of the rotation group is $2 N_{1}$.

The rotation group of the icosahedron (and therefore of its dual polyhedron, the dodecahedron) has 60 elements. 


\section{A.2 The invariants under discrete subgroups}

In this Section we will compute the lowest-degree homogeneous polynomials in the $(x, y, z)$ coordinates that are invariant under the action of several discrete subgroups of $O(3)$. We will consider only the subgroups which are really three-dimensional isometries: they are related to the Platonic solids discussed above. The other subgroups of proper rotations are the cyclic groups $C_{n}$, for $n>1$, and the dihedric groups $D_{n}$, for $n>2$, which are also subgroups of $\mathrm{SO}(2)$.

All invariants of $O(3)$ can be obtained as powers of the basic degree-two invariant

$$
I_{2}=x^{2}+y^{2}+z^{2}
$$

\section{A.2.1 The cubic group}

Let us fix the cube with vertices at the points $( \pm 1, \pm 1, \pm 1) / \sqrt{3}$. There are 48 matrices which leave invariant the cube, that are

$$
\begin{aligned}
& \left(\begin{array}{rrr} 
\pm 1 & 0 & 0 \\
0 & \pm 1 & 0 \\
0 & 0 & \pm 1
\end{array}\right), \quad\left(\begin{array}{rrr}
0 & 0 & \pm 1 \\
\pm 1 & 0 & 0 \\
0 & \pm 1 & 0
\end{array}\right), \quad\left(\begin{array}{rrr}
0 & \pm 1 & 0 \\
0 & 0 & \pm 1 \\
\pm 1 & 0 & 0
\end{array}\right), \\
& \left(\begin{array}{rrr} 
\pm 1 & 0 & 0 \\
0 & 0 & \pm 1 \\
0 & \pm 1 & 0
\end{array}\right), \quad\left(\begin{array}{rrr}
0 & \pm 1 & 0 \\
\pm 1 & 0 & 0 \\
0 & 0 & \pm 1
\end{array}\right), \quad\left(\begin{array}{rrr}
0 & 0 & \pm 1 \\
0 & \pm 1 & 0 \\
\pm 1 & 0 & 0
\end{array}\right) .
\end{aligned}
$$

Twenty-four matrices $\left(2 N_{1}=24\right)$ are proper rotations, while the other 24 matrices are obtained by compositions of proper rotations with the antipodal transformation $\operatorname{diag}(-1,-1,-1)$. Algebraically, the cubic group is $S_{4} \otimes \mathbb{Z}_{2}$, where $S_{4}$ is the group of permutations of 4 elements. As the cube is dual to the octahedron the two groups of invariance are the same.

It is easy to see that the lowest-order non-trivial polynomial is

$$
I_{4}=x^{4}+y^{4}+z^{4}+a I_{2}^{2} .
$$

On the unit sphere the polynomials can be decomposed into irreducible representations of $\mathrm{O}(3)$, i.e. in terms of spherical harmonics. Then

$$
I_{4}=\left(\frac{3}{5}+a\right) \sqrt{4 \pi} Y_{0,0}+\frac{2}{15} \sqrt{4 \pi} Y_{4,0}+\frac{2}{3} \sqrt{\frac{4 \pi}{70}}\left(Y_{4,4}+Y_{4,-4}\right) \text {. }
$$

With the choice $a=-3 / 5$, we obtain an operator that is renormalized multiplicatively.

\section{A.2.2 The group of the tetrahedron}

Let us choose the tetrahedron with vertices at the points

$$
\frac{1}{\sqrt{3}}(1,1,1), \frac{1}{\sqrt{3}}(1,-1,-1), \frac{1}{\sqrt{3}}(-1,1,-1), \frac{1}{\sqrt{3}}(-1,-1,1) .
$$

The matrices which leave invariant the tetrahedron are the 24 matrices of the cubic group which have an even number of -1 . Remark that there is not the antipodal 
transformation, but there are 12 proper rotations and 12 reflections. The lowest nontrivial polynomial is

$$
I_{3}=x y z=-i \sqrt{\frac{2 \pi}{105}}\left(Y_{3,2}-Y_{3,-2}\right) .
$$

Algebraically this group is $A_{4}$, the group of even permutations of 4 elements.

\section{A.2.3 The group of the icosahedron}

We shall parametrize the 12 vertices of the icosahedron as follows:

$$
\begin{aligned}
& P_{u}=(0,0,1), \\
& P_{d}=(0,0,-1), \\
& P_{k}=\left(\frac{2}{\sqrt{5}} \cos \left(\frac{\pi k}{5}\right), \frac{2}{\sqrt{5}} \sin \left(\frac{\pi k}{5}\right), \frac{1}{\sqrt{5}} \cos (k \pi)\right),
\end{aligned}
$$

for $k=1, \cdots, 10$. In order to construct invariant polynomials under the rotation group of the icosahedron, we first consider the cyclic group of order 5 of rotations of $2 \pi / 5$ around the $z$-axis. On the vertices it acts as a permutation of the form

$$
\left(P_{u}\right)\left(P_{1} P_{3} P_{5} P_{7} P_{9}\right)\left(P_{2} P_{4} P_{6} P_{8} P_{10}\right)\left(P_{d}\right) .
$$

In order to determine the invariants under this cyclic group, it is convenient to use cylindric coordinates $(z, \rho, \phi) \in R \times R^{+} \times[0,2 \pi]$ so that the action of the generator is

$$
(z, \rho, \phi) \rightarrow\left(z, \rho, \phi+\frac{2 \pi}{5}\right)
$$

and thus the invariants are $z, \rho$ and $5 \phi$. The lowest-order non-trivial polynomial which also respect the invariance under the antipodal transformation is of sixth degree and has the general form

$$
I_{6}=z\left(z^{5}+a z^{3} \rho^{2}+b z \rho^{4}+c \rho^{5} \cos (5 \phi)+d \rho^{5} \sin (5 \phi)\right)+e I_{2}^{3},
$$

where we have fixed to one the coefficient of $z^{6}$. Of course, $I_{6}$ must be the same on all the vertices of the icosahedron: this gives the condition

$$
a=31-4 b-8 c .
$$

We shall then use the cyclic group of order 3 of rotations around the axis which joins the origin with the center of the face $P_{u} P_{2} P_{4}$. On the vertices it acts as a permutation:

$$
\left(P_{u} P_{2} P_{4}\right)\left(P_{1} P_{5} P_{8}\right)\left(P_{3} P_{6} P_{10}\right)\left(P_{7} P_{9} P_{d}\right) .
$$

It gives the conditions

$$
\begin{aligned}
& b=5, \\
& c=2, \\
& d=0,
\end{aligned}
$$

so that $a=-5$. We obtain finally

$$
I_{6}=z\left(z^{5}-5 z^{3} \rho^{2}+5 z \rho^{4}+2 \rho^{5} \cos (5 \phi)\right)+e\left(z^{2}+\rho^{2}\right)^{3}
$$


or, in Cartesian coordinates,

$$
\begin{aligned}
I_{6}= & z\left(z^{5}-5 z^{3}\left(x^{2}+y^{2}\right)+5 z\left(x^{2}+y^{2}\right)^{2}+2 x\left(x^{4}-10 x^{2} y^{2}+5 y^{4}\right)\right) \\
& +e\left(x^{2}+y^{2}+z^{2}\right)^{3} .
\end{aligned}
$$

It can be checked that the all the other transformations, like the cyclic groups of order 2 around the center of an edge, for example the transformation which acts as a permutation like

$$
\left(P_{u} P_{2}\right)\left(P_{1} P_{6}\right)\left(P_{3} P_{8}\right)\left(P_{4} P_{10}\right)\left(P_{5} P_{9}\right)\left(P_{7} P_{d}\right)
$$

leave $I_{6}$ invariant. Also the antipodal transformation leaves $I_{6}$ invariant.

On the unit sphere, in terms of spherical coordinates, we obtain

$$
I_{6}=\left(\frac{5}{21}+e\right) \sqrt{4 \pi} Y_{0,0}+\frac{16}{21} \sqrt{\frac{4 \pi}{13}} Y_{6,0}-\frac{16}{3} \sqrt{\frac{4 \pi}{1001}}\left(Y_{6,5}-Y_{6,-5}\right)
$$

Therefore, for

$$
e=-\frac{5}{21}
$$

we obtain an operator which is multiplicatively renormalized.

Of course we could use a different position of the icosahedron in space, for example we could take for the vertices

$$
\frac{1}{\sqrt{2+\tau}}(0, \pm \tau, \pm 1) \quad \frac{1}{\sqrt{2+\tau}}( \pm \tau, \pm 1,0) \quad \frac{1}{\sqrt{2+\tau}}( \pm 1,0, \pm \tau)
$$

where $\tau$ is golden ratio. In this basis the cyclic group of rotations of order five is generated by

$$
\frac{1}{2}\left(\begin{array}{ccc}
\tau & \tau-1 & -1 \\
\tau-1 & 1 & \tau \\
1 & -\tau & \tau-1
\end{array}\right)
$$

The new choice can be recovered from the old one by a rotation and this produces a different polynomial which turns out to be

$$
\begin{aligned}
I_{6}^{\prime}= & \left(\frac{5}{21}+e\right) \sqrt{4 \pi} Y_{0,0}-5 \sqrt{\frac{4 \pi}{273}}\left[\frac{1}{\sqrt{21}} Y_{6,0}-\frac{1}{2}\left(Y_{6,2}+Y_{6,-2}\right)\right. \\
& \left.-\frac{1}{\sqrt{6}}\left(Y_{6,4}+Y_{6,-4}\right)+\frac{1}{2} \sqrt{\frac{5}{11}}\left(Y_{6,6}+Y_{6,-6}\right)\right],
\end{aligned}
$$

which, of course, with the choice (39) belongs to the same multiplet $l=6$.

The icosahedron is dual to the dodecahedron and thus their group of invariance is the same.

\section{B The perturbative results}

In [20] the anomalous dimension of all non-derivative dimension-zero operators was computed for the nearest-neighbor lattice $O(N) \sigma$-model up to three loops. For generic $N$, a suitable basis for these operators is given by

$$
\mathcal{O}_{j_{1} \ldots j_{n}}^{(n)}=\boldsymbol{\sigma}^{j_{1}} \ldots \boldsymbol{\sigma}^{j_{n}}-\text { traces }
$$


where "traces" must be such that $\mathcal{O}_{j_{1} \ldots j_{n}}^{(n)}$ is completely symmetric and traceless. These polynomials are irreducible $O(N)$-tensors of rank $n$ and as such they renormalize multiplicatively with no off-diagonal mixing. For $N=3$ this representation is equivalent to that of the spherical harmonics.

The anomalous dimension $\gamma^{(n)}(t)$ of these operators is given by

$$
\gamma^{(n)}(t)=\gamma_{0}^{(n)} t+\gamma_{1}^{(n)} t^{2}+\gamma_{2}^{(n)} t^{3}+O\left(t^{4}\right)
$$

where

$$
\begin{aligned}
\gamma_{0}^{(n)} & =\frac{n(N+n-2)}{4 \pi}, \\
\gamma_{1}^{(n)} & =\frac{n(N+n-2)}{16 \pi}, \\
\gamma_{2}^{(n)} & =\frac{n(N+n-2)}{4 \pi}\left[\frac{N-2}{4 \pi^{2}}\left(4 \pi^{2} G_{1}+\frac{1}{2}-\frac{\pi^{2}}{8}\right)+\frac{11}{96}\right],
\end{aligned}
$$

where

$$
G_{1}=-\frac{1}{4} \int_{[-\pi, \pi]^{d}} \frac{\mathrm{d}^{d} p}{(2 \pi)^{d}} \frac{\mathrm{d}^{d} q}{(2 \pi)^{d}}\left[\sum_{\mu}\left(\widehat{(p+q)^{4}}\right] \frac{(\widehat{p+q})^{2}-\hat{p}^{2}-\hat{q}^{2}}{\hat{p}^{2} \hat{q}^{2}\left[(\widehat{p+q})^{2}\right]^{2}}\right.
$$

and

$$
\hat{p}^{2}=\sum_{\mu} \hat{p}_{\mu}^{2}=\sum_{\mu}\left(2 \sin \frac{p_{\mu}}{2}\right)^{2} .
$$

Numerically $G_{1} \approx 0.0461636$. For $N=3$ we obtain

$$
\begin{aligned}
& \gamma^{(3)}(t)=0.95493 t+0.238732 t^{2}+0.135755 t^{3} \\
& \gamma^{(4)}(t)=1.59155 t+0.397887 t^{2}+0.226258 t^{3} \\
& \gamma^{(6)}(t)=3.34225 t+0.835563 t^{2}+0.475142 t^{3}
\end{aligned}
$$




\section{References}

[1] S. Elitzur, R. B. Pearson, and J. Shigemitsu, Phys. Rev. D19 (1978) 3698.

[2] C. Rebbi, Phys. Rev. D21 (1980) 3350.

[3] G. Bhanot and C. Rebbi, Phys. Rev. D24 (1981) 3319.

[4] J. V. José, L. P. Kadanoff, S. Kirkpatrick, and D. R. Nelson, Phys. Rev. B16 (1977) 1217.

[5] J. Fröhlich and T. Spencer, Comm. Math. Phys. 81 (1981) 527.

[6] A. Patrascioiu and E. Seiler, Phys. Lett. B430 (1998) 314 hep-lat/9706011.

[7] A. Patrascioiu and E. Seiler, Phys. Lett. B445 (1998) 160 hep-th/9809154.

[8] A. Patrascioiu and E. Seiler, hep-th/0002153.

[9] A. Patrascioiu and E. Seiler, hep-lat/0008024.

[10] P. Hasenfratz and F. Niedermayer, Nucl. Phys. B596 (2001) 481 hep-lat/0006021.

[11] P. Hasenfratz and F. Niedermayer, Nucl. Phys. B (Proc. Suppl.) 94 (2001) 575. hep-lat/0011056.

[12] S. Caracciolo, R. G. Edwards, A. Pelissetto, and A. D. Sokal, Phys. Rev. Lett. 75 (1995) 1891 hep-lat/9411009.

[13] S. Caracciolo, R. G. Edwards, S. J. Ferreira, A. Pelissetto, and A. D. Sokal, Phys. Rev. Lett. 74 (1995) 2969 hep-lat/9409004.

[14] R. A. Pelcovits and D. R. Nelson, Phys. Lett. 57A (1976) 23.

[15] J. M. Kosterlitz, D. R. Nelson, and M. E. Fisher, Phys. Rev. B 13 (1976) 412.

[16] D. J. Amit, "Field Theory, the Renormalization Group, and Critical Phenomena," Ch. 5-3 Part II (World Scientific, Singapore, 1984).

[17] J. Zinn-Justin, "Quantum Field Theory and Critical Phenomena," third edition with corrections (Clarendon, Oxford, 1997).

[18] A. Patrascioiu, J. L. Richard and E. Seiler, Phys. Lett. B 254 (1991) 173.

[19] H. S. M. Coxeter, Regular Polytopes (Dover, New York, 1973).

[20] S. Caracciolo and A. Pelissetto, Nucl. Phys. B420 (1994) 141 hep-lat/9401015. 